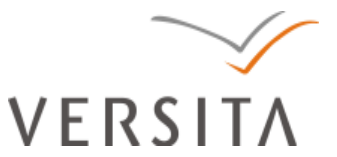

\title{
BIFURCATION, CHAOS AND ATTRACTOR \\ IN THE LOGISTIC COMPETITION
}

Małgorzata Guzowska, Ph.D.

Department of Econometrics and Statistics

Faculty of Economics and Management

University of Szczecin

Mickiewicza 64, 71-101 Szczecin

e-mail: mguzowska@wneiz.pl

Received 13 September 2011, Accepted 11 February 2012

\begin{abstract}
This paper deals with a two- dimensional discrete time competition model. The corresponding twodimensional iterative map is represented in terms of its bifurcation diagram in the parameter plane. A number of bifurcation sequences for attractors and their basins are studied.
\end{abstract}

Keywords: Discrete Logistic Competition Model, local stability, global stability, bifurcation, chaos, chaotic attractor.

JEL classification: C02, C15, C62. 


\section{Introduction}

In 1798 Malthus proposed the population growth model $^{1}$ having observed that the world population grew geometrically, while food resources grew arithmetically. Verhulst, however, conditioned the changes in population on limited natural resources, such as land and food, etc., At the same time he introduced the term of carrying capacity due to which his model became the first competition model which he himself called a logistic model ${ }^{2}$. Over one hundred years later, in 1976, a biologist Robert $\mathrm{May}^{3}$ used for the first time the discreet model of the population growth. It became the starting point of the chaos theory when the researchers started using the logistic model being a discreet version of the Verhulst model to describe the evolution of the population of various species (e.g. in ecology or demography) or corporation growth in economics ${ }^{4}$.

Simultaneously, in the 1920s Lotka and Volterra ${ }^{5}$ proposed a model (in continuous time) describing mutual impact of two species, each of which, if left isolated, would be subject to the logistic growth. They assumed that if both species existed simultaneously, competition appeared that hindered their population density. On the basis of those first competition models new models were developed, and are still being developed, employing both differential and difference equations. Models of this form are often used for the description of biological, social or economic ${ }^{6}$ systems where two individuals, or two populations, compete in the environment where they are operating. For example, in economic modeling model variables may represent choices (concerning e.g. production or investment) of two firms which produce the same goods and compete in order to maximize their profits by acquiring greater portions of a given market.

\section{Model and its local stability properties}

In this section we provide a brief review of the results presented by Guzowska, Luis and Elaydi ${ }^{\mathrm{g}}$. In this paper ${ }^{\mathrm{h}}$ authors considered the following logistic competition model:

$$
\left\{\begin{array}{l}
x_{n+1}=\frac{a x_{n}\left(1-x_{n}\right)}{1+c y_{n}} \\
y_{n+1}=\frac{b y_{n}\left(1-y_{n}\right)}{1+d x_{n}}
\end{array}\right.
$$

where $x, y \in[0,1], a, b>0$ (the intrinsic growth rates) and $c, d \in(0,1)$ (the competition parameters). The map associated with (1) is given by: 


$$
F(x, y)=\left(\frac{a x(1-x)}{1+c y}, \frac{b y(1-y)}{1+d x}\right)
$$

The map $F$ has one fixed point $(0,0)$, two exclusion fixed points $((a-1) / a, 0),(0,(b-1) / b)$ as well as one coexistence fixed point:

$$
\left(x^{*}, y^{*}\right)=\left(\frac{-c b+a b-b+c}{a b-c d}, \frac{-a d+a b-a+d}{a b-c d}\right) .
$$

The Jacobian matrix of the map $F$ of the system (1) is presented in the form of:

$$
F(x, y)=\left[\begin{array}{cc}
\frac{a(1-x)-a x}{1+c y} & \frac{-a c x(1-x)}{(1+c y)^{2}} \\
\frac{-b d y(1-y)}{(1+d x)^{2}} & \frac{b(1-y)-b y}{1+d x}
\end{array}\right] .
$$

In the point $(0,0)$ the Jacobian takes the form:

$$
J_{0}=J F(0,0)=\left[\begin{array}{ll}
a & 0 \\
0 & b
\end{array}\right] .
$$

If $a \leq 1$ and $b \leq 1$, then, according to Lemma $1^{\mathrm{i}}$, the point $(0,0)$ is a globally asymptotically stable fixed point. If $a>1$ or $b<1$, the point $(0,0)$ becomes unstable.

For the point $((a-1) / a, 0)$ the Jacobian takes the form:

$$
J_{a}=J F\left(\frac{a-1}{a}, 0\right)=\left[\begin{array}{cc}
2-a & \frac{-c(a-1)}{a} \\
0 & \frac{a b}{a d+a-d}
\end{array}\right] .
$$

Eigenvalues of the matrix $J_{a}$ to $\lambda_{1}=2-a$ and $\lambda_{2}=a b /(a d+\mathrm{a}-d)$. Thus the point $((a-1) / a, 0)$ is an asymptotically stable fixed point for $1<a<3$ and $1<b<1+d(a-1) / a$. When $a=3$ the fixed point $((a-1) / a, 0)$ is asymptotically stable, while when $1<a<3$ and $b=1+d(a-1) / a$ it is unstable.

Analogous properties are observed for the point $(0,(b-1) / b)$.

The coexistence fixed point:

$$
\left(x^{*}, y^{*}\right)=\left(\frac{-c b+a b-b+c}{a b-c d}, \frac{-a d+a b-a+d}{a b-c d}\right),
$$


exists only if the system parameters satisfy the following inequalities:

$$
\frac{a-1}{c}>\frac{b-1}{b} \text { and } \frac{b-1}{d}>\frac{a-1}{a} \text {. }
$$

The Jacobian for the fixed point $\left(x^{*}, y^{*}\right)$ takes the form of:

$$
J^{*}=J F\left(x^{*}, y^{*}\right)=\left[\begin{array}{cc}
\frac{1-2 x^{*}}{1-x^{*}} & \frac{-c x^{*}}{a\left(1-x^{*}\right.} \\
\frac{-d y^{*}}{b\left(1-y^{*}\right)} & \frac{1-2 y^{*}}{1-y^{*}}
\end{array}\right] .
$$

The point $\left(x^{*}, y^{*}\right)$ will be locally asymptotically stable if the modules of all the Jacobian eigenvalues will be less than one. If $P(\lambda)$ is a characteristic equation of the Jacobian $J^{*}$, according to Jury test ${ }^{10}$, the eigenvalues of the Jacobian $J^{*}$ are inside the unit, if only if the following inequalities are satisfied:

$$
\text { (i) } P(1)>0, \quad \text { (ii) } P(-1)>0, \quad \text { (iii) }|P(0)|<1 \text {. }
$$

In the paper by Guzowska, Luis and Elaydi ${ }^{11}$ it is proved that $\left(x^{*}, y^{*}\right)$ is an asymptotically stable fixed point, when $1<a<3$ and $1<b<3$.

\section{Bifurcation, chaos and attractors}

I start the analysis of the examined model properties from the description of the parameter plane properties. The tool that permits indicating the combinations of parameters in the presence of which the examined system has stabile attractors is a two-dimensional bifurcation diagram. The two-dimensional bifurcation diagrams allow for defining these parameter values, in the presence of which the dynamical system has periodic and quasi-periodic attractors.

In the works by Elaydi ${ }^{12}$ and Kuznetsov ${ }^{13}$ the authors present the analysis of three main types of the bifurcations of two-dimensional discreet dynamical systems. They include saddlenode bifurcation existing when one of the Jacobian eigenvalues equals 1 (when we cross the line $\operatorname{det}\left(J^{*}\right)=\operatorname{tr}\left(J^{*}\right)-1$ starting from a stable region). The second type is the period-doubling bifurcation which exists when one the Jacobian eigenvalues equals -1 (on the basis of the Jacobian trace and determinant analysis it is the case when we cross the line $\operatorname{det}\left(J^{*}\right)=-\operatorname{tr}\left(J^{*}\right)-1$ starting from a stable region). And finally, the third type is the Neimark - Sacker bifurcation present when the Jacobian eigenvalues are complex numbers and their modules equal 1 (when $\operatorname{det}\left(J^{*}\right)=1$ and $\left.-2<\operatorname{tr}\left(J^{*}\right)<2\right)$. 
The coexistence equilibrium point $\left(x^{*}, y^{*}\right)$ is asymptotically stable when the modules of the Jacobian eigenvalues are less than 1 . These conditions will be satisfied when the following inequalities are satisfied as well ${ }^{14}$ :

$$
\left|\operatorname{tr}\left(J^{*}\right)\right|-1<\operatorname{det}\left(J^{*}\right)<1
$$

If any of the three above inequalities is not satisfied, the fixed point is not stable.

The computations prove that the inequality $\operatorname{det}\left(J^{*}\right)<1$ takes form:

$$
\begin{gathered}
\left(\frac{-c(b-c+b c) d^{2}+a^{3} b^{2}(2-b+2 d)+a(b-c+b c) d(3 b+c+c d)}{a b(-(1+c) d+a(1+d))(-b(1+c)+c(1+d))}+\right. \\
\left.+\frac{a^{2} b\left(2 b^{2}(1+c)+3 c(1+d)-b(3+5 d+c(5+4 d))\right)}{a b(-(1+c) d+a(1+d))(-b(1+c)+c(1+d))}\right)<0 .
\end{gathered}
$$

The inequality $\operatorname{det}\left(J^{*}\right)<\operatorname{tr}\left(J^{*}\right)-1$ takes form:

$$
\frac{b(-1+a-c)+c)(a(-1+b-d)+d)(a b-c d)}{a b(-(1+c) d+a(1+d))(-b(1+c)+c(1+d))}<0 .
$$

While the inequality $\operatorname{det}\left(J^{*}\right)>-\operatorname{tr}\left(J^{*}\right)-1$ takes form:

$$
\begin{gathered}
\left(\frac{-c(b-c+b c) d^{2}+a^{3} b^{2}(3-b+3 d)}{a b(-(1+c) d+a(1+d))(-b(1+c)+c(1+d))}+\right. \\
+\frac{-a d\left(-b^{2}\left(9+14 c+5 c^{2}\right)+c^{2}(1+d)+b c(8+4 c+4 d+3 c d)\right)}{a b(-(1+c) d+a(1+d))(-b(1+c)+c(1+d))}+ \\
\left.+\frac{a^{2} b\left(3 b^{2}(1+c)+c\left(9+14 d+5 d^{2}\right)-3 b(3+4 d+4 c(1+d))\right)}{a b(-(1+c) d+a(1+d))(-b(1+c)+c(1+d))}\right)>0 .
\end{gathered}
$$

Basing on the above three inequalities, as well as using the fundamental bifurcation theory, we can present in a graphical form the bifurcation scenario in the two-dimensional parameter plane $(a, b)$ when parameters $\mathrm{c}$ and $\mathrm{d}$ have fixed values (Figure 1).

The stability region of the coexistence equilibrium point in the parameter plane $(a, b)$ is shown as the region $S_{1}$ in Figure 1. This is the region between the curves $\gamma_{1}$ and $\gamma_{2}$ defined by:

$$
\begin{aligned}
& \gamma_{1}=\left\{(a, b) \in R_{+}^{2}: b=1+d\left(\frac{a-1}{a}\right)\right\}, \\
& \gamma_{2}=\left\{(a, b) \in R_{+}^{2}: a=1+c\left(\frac{b-1}{b}\right)\right\} .
\end{aligned}
$$




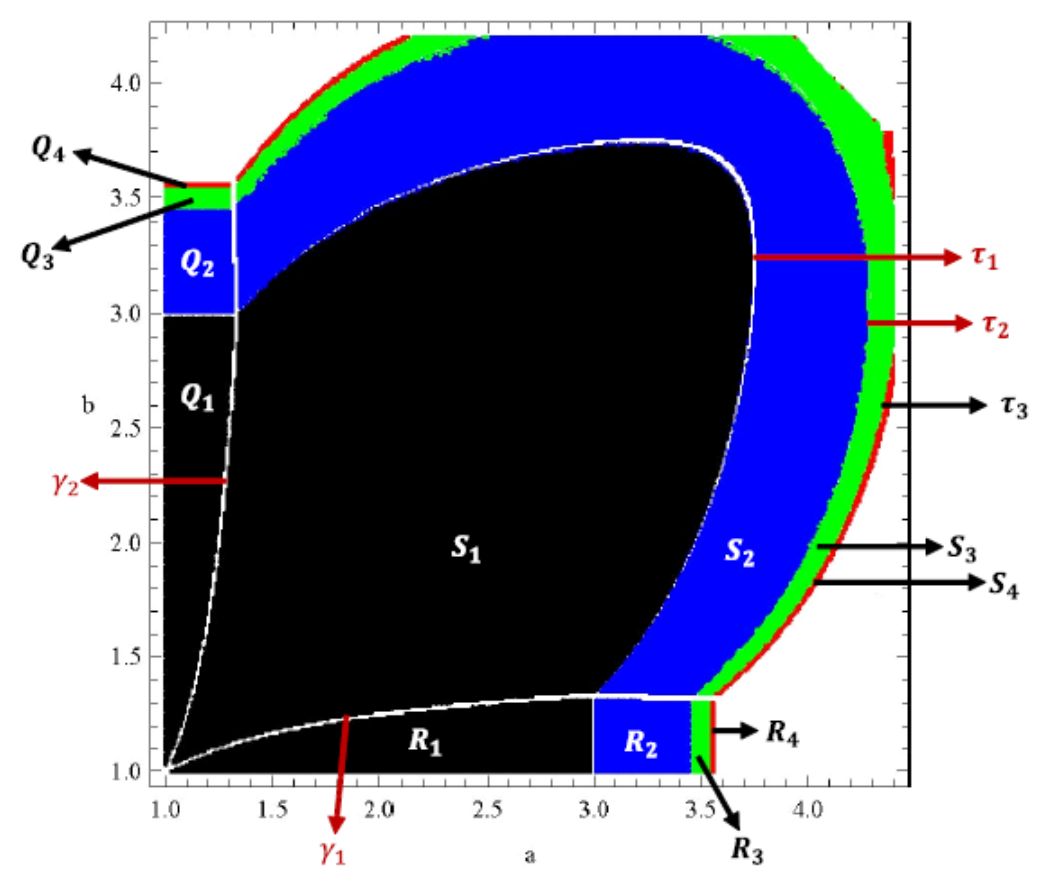

Fig. 1. The stability regions and bifurcation scenario of the logistic competition model in the parameter plane $(a, b)$ for fixed $c=d=0.5$. The Figure presents only the orbits of the periods 1, 2, 4 and 8)

Source: own computations and study with the use of the Mathematica software.

$S_{1}$ is the stability region of the coexistence equilibrium point $\left(x^{*}, y^{*}\right), R_{1}$ is the stability region of the exclusion fixed point on the $x$-axis $((a-1) / a, 0)$, and $Q_{1}$ is the stability region of the exclusion fixed point on the $y$-axis $(0,(b-1) / b)$. A period-doubling bifurcation results (in the coexistence case) as we move from $S_{1}$ to $S_{2}$, to $S_{3}$, etc. Similarly, we observe a perioddoubling scenario (in the exclusion case) on the $x$ - axis as we move from $R_{1}$ to $R_{2}$, to $R_{3}$, etc. And again, we observe a period-doubling scenerio (in the exclusion case) in the $y$-axis as we move from $Q_{1}$ to $Q_{2}$, to $Q_{3}$, etc.

Figure 2 shows the extended two-dimensional bifurcation diagram in the $(a, b)$ parameter plane. The combinations of parameters for which the system (1) demonstrates stable cycles of the period less than 34 are marked with colours. The dominating dynamics of the system (1) is a cyclic dynamics of short periods. Subsequent attractors are marked with the following colours: red - the region with asymptotically stable convergence into one of the fixed points; blue - the 
emergence of the cycles of period 2; yellow - the cycles of period 4; cream - cycles of period 8 , etc. up to the cycles of period 35 . The white area in the diagram represents the cycles of periods larger than 34 or with the quasi-periodic attractor, while black denotes instability (for these parameter values the model does not have any attractor).

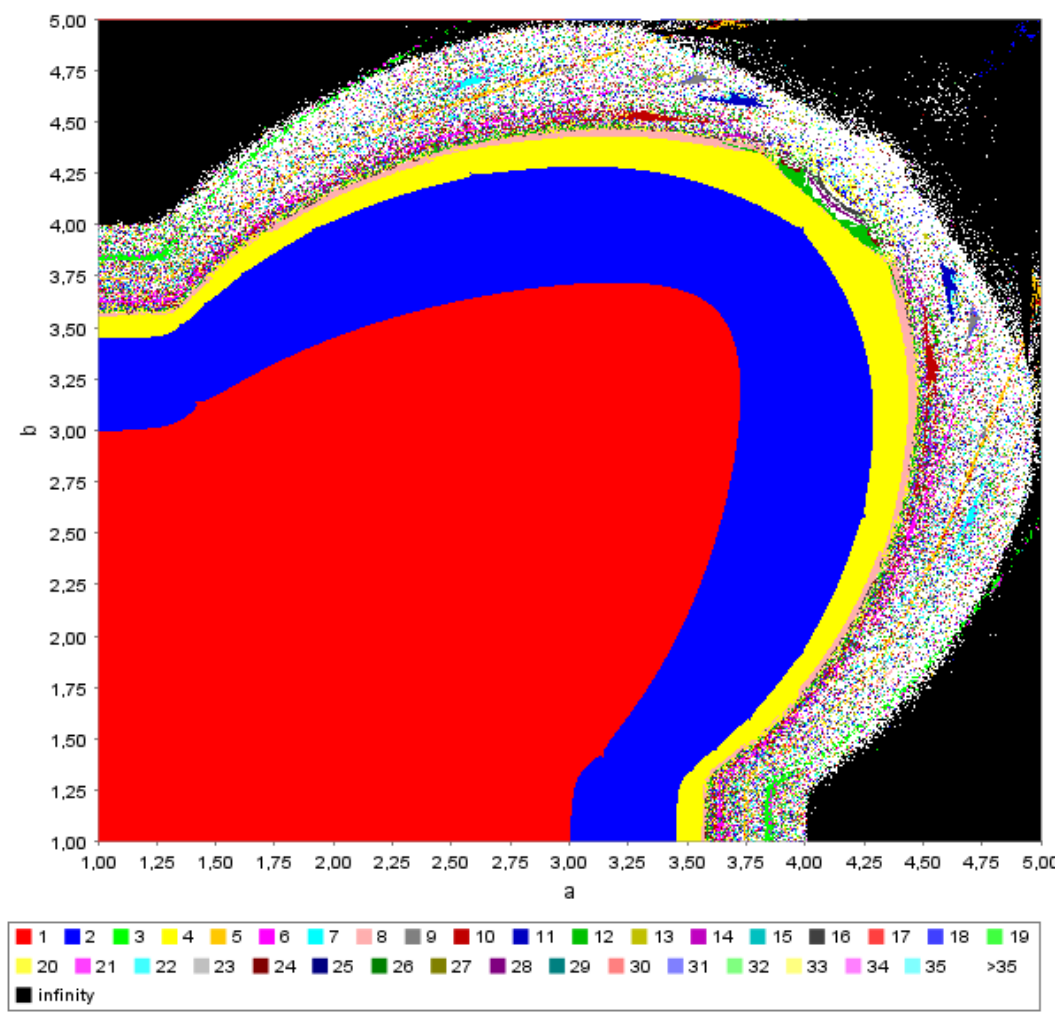

Fig. 2. The two-dimensional bifurcation diagram of the map (1) in the $(a, b)$ - parameter plane where the existing regions of attracting cycles of the period $k, k<32$, are shown $(c=d=0.5)$

Source: own computations and study with the use of the iDMC software

The above scenario can be confirmed by the largest Lyapunov exponent analysis. Figure 3 presents a bifurcation diagram along with the largest Lyapunov exponents according to the change of a parameter with $a$ fixed value $b$ (e.g. in Figure 3, $b=4$ ). The behaviour of both the bifurcation diagram and the largest Lyapunov exponents confirm the above scenario. 
Additionally, Figure 4 shows globally the properties of the largest Lyapunov exponents for the system (1) depending on the changes in the parameters a and $b$. There are 6 potential scenarios according to the sign of the exponents as well as to their zero value. If one of the Lyapunov exponents exceeds zero towards plus values, it means that the system behaves chaotically.
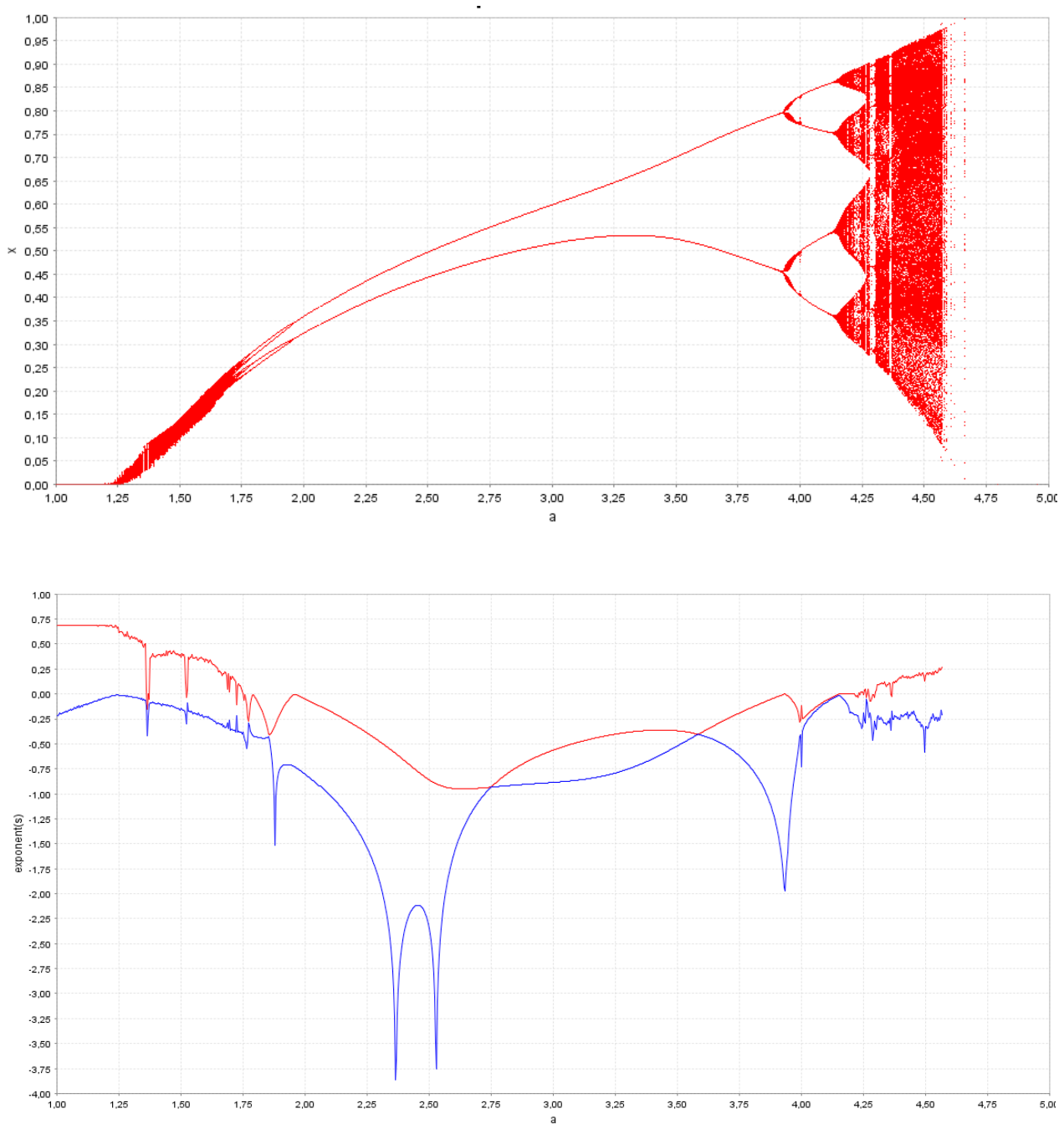

Fig. 3. The bifurcation diagram of the logistic competition model with the Lyapunov exponents for the parameter a with the fixed parameter value $b=4$

Source: own computations and study with the use of the Mathematica software. 
The analysis of the bifurcation diagrams, also the ones presented in Figures 2 and 3, as well as additional computations allow for spotting in the model not only the periodic attractors or chaos, but also the chaotic attractors. In case of the logistic competition model it is apparent (see Figure 3 ) that the area of chaotic dynamics is intersected with the so-called windows of stable periodic attractors. Then, along with the growth of the parameter $a$ only the chaotic attractor exists. The evolution of the attractors for four values of the parameter a is presented in Figure 5.

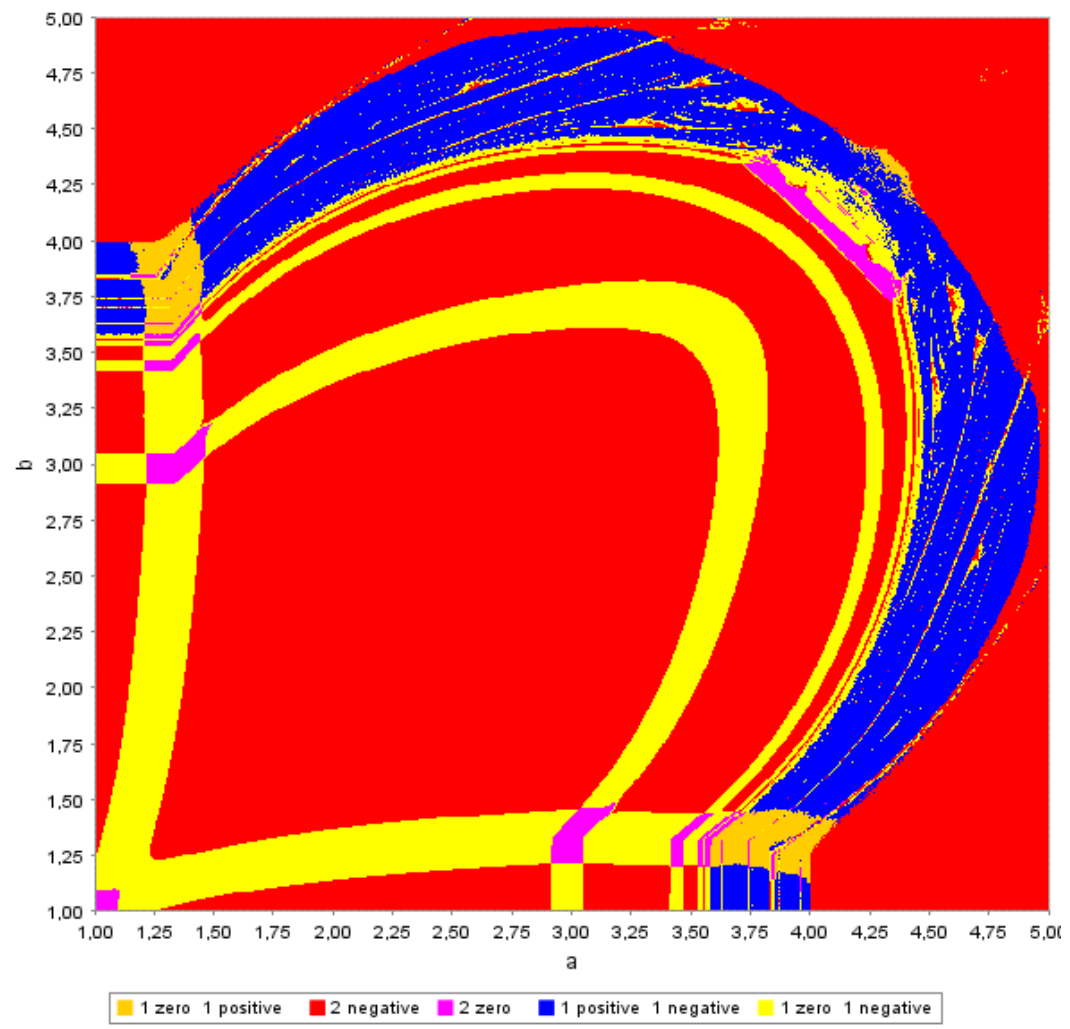

Fig. 4. The largest Lyapunov exponents for the system (1) depending on the changes of the parameters $a$ and $b$

Source: own computations and study with the use of the iDMC software. 

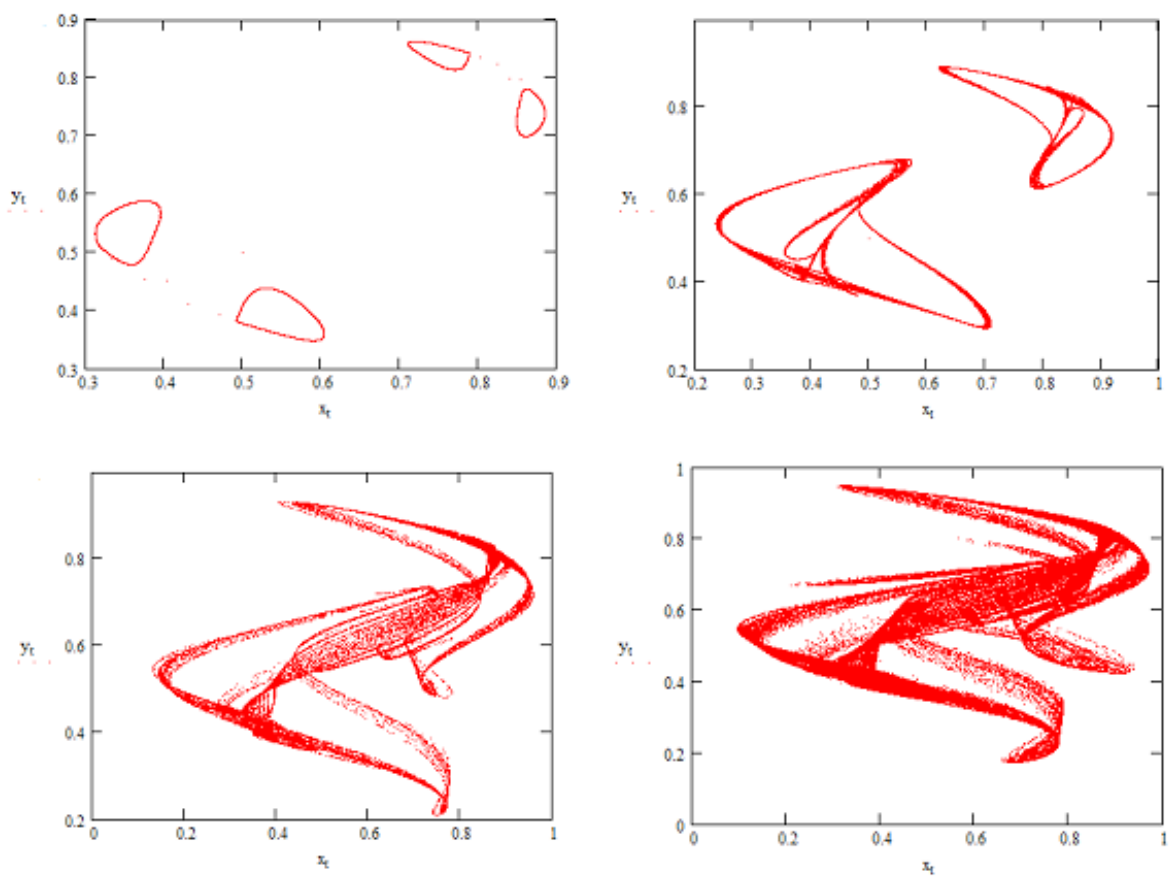

Fig. 5. The attractors of the logistic competition model for four different values of the parameter $a(a=4.2, a=4.35, a=4.5, a=4.55)$, when the remaining parameter values are $(c=d=0.5)$

Source: own computations and study with the use of the MathCad software.

\section{Conclusions}

The paper presents the dynamics of the logistic competition model described by nonlinear difference equations. The author discusses the dynamic properties of the model according to the changes in the decision parameters and presents the bifurcation scenario in the $a$ and $b$ parameters plane. Additionally, the conditions for the presence and the design mechanism of two types of bifurcation (the saddle-node and the period doubling ones) are identified. Finally, the paper shows how chaotic behaviour and chaotic attractors emerge in the model.

The present study is not only the outline analysis of the global dynamics, of chaotic behaviour and of attractors emergence in the presented logistic competition model. In her further studies the author will deal with the application of the critical curves method ${ }^{15}$ as a tool for studying the global dynamical properties of two-dimensional noninvariable maps in order 
to understand better the bifurcation mechanism, the attractors emergence and, above all, the economic interpretation of such phenomena.

\section{Notes}

1 Malthus (1798).

2 Varhulst (1845).

3 May (1976).

4 Day (1994), Puu (1989), Puu (1991), Puu (2000).

Volterra (1928).

6 Day (1994), Bischi, Gardini (2000), López-Ruiz, Fournier-Prunaret (2004), Puu (2000).

Guzowska, Luis, Elaydi (2011).

Ibidem.

Ibidem.

10 Elaydi (1996).

11 Guzowska, Luis, Elaydi (2011).

12 Elaydi (2008).

13 Kuznetsov (1995).

14 See Elaydi (2008), p. 200.

15 Mira (1987), Mira, Gardini, Barugola, Cathala (1996).

\section{References}

Bischi, G.I., Gardini, L. (2000). Global Properties of Symmetric Competition Models with Riddling and Blowout Phenomena. Discrete Dynamics in Nature and Society. Vol. 5, 149160 .

Day, R.H. (1994). Complex Economic Dynamics. The MIT Press, Cambridge, Massachusetts.

Elaydi, S. (1996). An Introduction to Difference Equations. Springer, New York.

Elaydi, S. (2008). Discrete Chaos: With Applications in Science and Engineering. Chapman and $\mathrm{Hall} / \mathrm{CRC}$.

Guzowska, M., Luis, R., Elaydi, S. (2011). Bifurcation and invariant manifolds of the logistic competition model. Journal of Difference Equations and Applications. Vol. 17, Issue 12.

Kuznetsov, Y. (1995). Elements of applied bifurcation theory. Vol. 112 of Applied mathematical sciences, SV, New York . 
López-Ruiz, R., Fournier-Prunaret, D. (2004). Indirect Allee effect, bistability and chaotic oscillations in a predator-prey discrete model of logistic type. Chaos, Solitons and Fractals, 24, 85-101.

Malthus, T.R. (1798). An Essay on the Principle of Population. Printed for J. Johnson, in St. Paul's Church-Yard. London.

May, R.M. (1976). Simple mathematical models with very complicated dynamics. Nature 261, 459-467.

Mira, C. (1987). Chaotic Dynamics. World Scientific, Singapour.

Mira, C., Gardini, L., Barugola, A., Cathala, J.-C. (1996). Chaotic Dynamics in Two-Dimensional Noninvertible Maps. World Scientific Series on Nonlinear Science. Series A, Vol. 20.

Puu, T. (1989). Nonlinear economic dynamics. In: Lecture notes in economics and mathematical systems, Vol. 336. Springer-Verlag.

Puu, T. (1991). Chaos in business cycles. Chaos, Solitons, \& Fractals. 1,457-73.

Puu, T. (2000). Attractors, bifurcations, and chaos - nonlinear phenomena in economics. Springer-Verlag.

Verhulst, P.F. (1845). Recherches mathématiques sur la loi d'accroissement de la population. Nouveaux Mémoires de l'Académie Royale des Sciences et Belles-Lettres de Bruxelles 18,1-42.

Volterra, V. (1928). Variations and fluctuations of the number of individuals in animal species living together. J. Cons. Int. Explor. Mer. 3 3-51. 